This is an electronic reprint of the original article. This reprint may differ from the original in pagination and typographic detail.

Author(s): Chaudhuri, Saumyadip; Maasilta, Ilari

Title: $\quad$ Superconducting tantalum nitride-based normal metal-insulator-superconductor tunnel junctions

Year: $\quad 2014$

Version:

Please cite the original version:

Chaudhuri, S., \& Maasilta, I. (2014). Superconducting tantalum nitride-based normal metal-insulator-superconductor tunnel junctions. Applied Physics Letters, 104(12), Article 122601. https://doi.org/10.1063/1.4869563

All material supplied via JYX is protected by copyright and other intellectual property rights, and duplication or sale of all or part of any of the repository collections is not permitted, except that material may be duplicated by you for your research use or educational purposes in electronic or print form. You must obtain permission for any other use. Electronic or print copies may not be offered, whether for sale or otherwise to anyone who is not an authorised user. 


\section{AIP Appled phyiles Letters}

\section{Superconducting tantalum nitride-based normal metal-insulator-superconductor tunnel junctions}

S. Chaudhuri and I. J. Maasilta

Citation: Applied Physics Letters 104, 122601 (2014); doi: 10.1063/1.4869563

View online: http://dx.doi.org/10.1063/1.4869563

View Table of Contents: http://scitation.aip.org/content/aip/journal/apl/104/12?ver=pdfcov

Published by the AIP Publishing

\section{Articles you may be interested in}

Niobium nitride-based normal metal-insulator-superconductor tunnel junction microthermometer

Appl. Phys. Lett. 102, 132601 (2013); 10.1063/1.4800440

Sub-micron normal-metal/insulator/superconductor tunnel junction thermometer and cooler using $\mathrm{Nb}$ Appl. Phys. Lett. 101, 112601 (2012); 10.1063/1.4751355

Bolometric response in graphene based superconducting tunnel junctions

Appl. Phys. Lett. 100, 153507 (2012); 10.1063/1.3703117

Josephson tunneling properties in MgB 2 /AIN/NbN tunnel junctions

J. Appl. Phys. 92, 7369 (2002); 10.1063/1.1511827

Local trap spectroscopy in superconducting tunnel junctions

Appl. Phys. Lett. 78, 3654 (2001); 10.1063/1.1377624

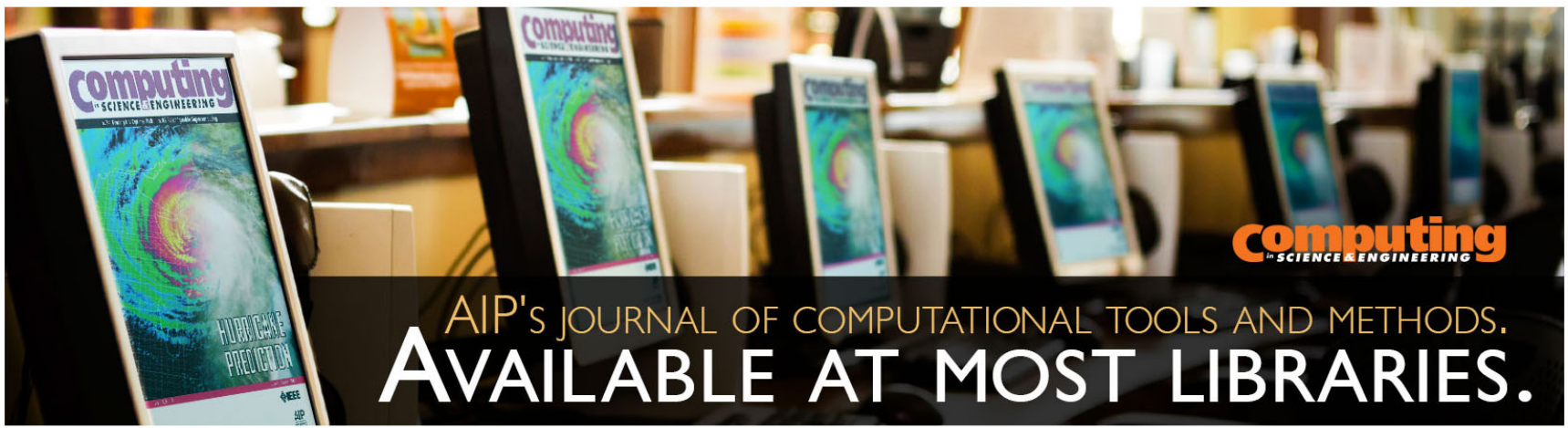




\title{
Superconducting tantalum nitride-based normal metal-insulator-superconductor tunnel junctions
}

\author{
S. Chaudhuri and I. J. Maasilta ${ }^{a}$ \\ Nanoscience Center, Department of Physics, University of Jyväskylä, P.O. Box 35, FIN-40014 Jyväskylä, \\ Finland
}

(Received 18 December 2013; accepted 13 March 2014; published online 26 March 2014)

\begin{abstract}
We report the development of superconducting tantalum nitride $\left(\mathrm{TaN}_{x}\right)$ normal metal-insulator-superconductor (NIS) tunnel junctions. For the insulating barrier, we used both $\mathrm{AlO}_{x}$ and $\mathrm{TaO}_{x}\left(\mathrm{Cu}-\mathrm{AlO}_{x}-\mathrm{Al}-\mathrm{TaN}_{x}\right.$ and $\left.\mathrm{Cu}-\mathrm{TaO}_{x}-\mathrm{TaN}_{x}\right)$, with both devices exhibiting temperature dependent current-voltage characteristics which follow the simple one-particle tunneling model. The superconducting gap follows a BCS type temperature dependence, rendering these devices suitable for sensitive thermometry and bolometry from the superconducting transition temperature $T_{\mathrm{C}}$ of the $\mathrm{TaN}_{x}$ film at $\sim 5 \mathrm{~K}$ down to $\sim 0.5 \mathrm{~K}$. Numerical simulations were also performed to predict how junction parameters should be tuned to achieve electronic cooling at temperatures above 1 K. (C) 2014 AIP Publishing LLC. [http://dx.doi.org/10.1063/1.4869563]
\end{abstract}

Normal metal-insulator-superconductor (NIS) tunnel junction devices aimed at low temperature thermometry, bolometry, and refrigeration have witnessed significant developments in the last decade. ${ }^{1,2}$ Aluminum (Al) based NIS devices already offer sensitive thermometry in the sub $1 \mathrm{~K}$ range, ${ }^{3}$ and significant cooling power approaching $1 \mathrm{nW}$ at $\sim 0.3 \mathrm{~K}$ has recently been demonstrated. ${ }^{4,5}$ In addition to direct electronic cooling, sizeable indirect phonon cooling of suspended membranes, ${ }^{6-8}$ beams, ${ }^{9}$ and a general-purpose refrigeration platform ${ }^{4}$ have been achieved using $\mathrm{Al}$ coolers. However, to achieve operation at higher temperatures, one must switch to materials with higher superconducting transition temperatures $\left(T_{\mathrm{C}}\right)$ than the $T_{\mathrm{C}}$ of $\mathrm{Al}$ at $\sim 1.2 \mathrm{~K}$, as $T_{\mathrm{C}}$ limits the maximum range of thermometry, and cooling power drops strongly above $T \sim 0.4 T_{\mathrm{C}}{ }^{1}$

Recently, we fabricated $\mathrm{Nb}\left(T_{\mathrm{C}} \sim 8 \mathrm{~K}\right)^{10}$ and $\mathrm{NbN}_{x}\left(T_{\mathrm{C}}\right.$ $\sim 12 \mathrm{~K})^{11}$ based NIS devices and demonstrated an order of magnitude increase of the thermometry range, and an observation of some electronic cooling in the $\mathrm{Nb}$ device. ${ }^{10}$ However, as the optimal operational temperature for cooling for those type of devices is around $3.5-5 \mathrm{~K}$, one should also develop NIS devices with a $T_{\mathrm{C}}$ in the intermediate range between $\mathrm{Al}$ and $\mathrm{Nb} / \mathrm{NbN}$ devices. This is important because the cooling power also deteriorates fast when the operational temperature is lower than the optimal, and thus $\mathrm{Nb}$ or $\mathrm{NbN}$ based coolers may not be able to work effectively enough in the temperature range $1-3 \mathrm{~K}$. Here, we experimentally demonstrate that tantalum nitride $(\mathrm{TaN})$ with a $T_{\mathrm{C}} \sim 5 \mathrm{~K}$ can be used as the superconducting electrode in a micron-scale NIS device. The thermometric characteristics were essentially ideal in the temperature range $0.5-5 \mathrm{~K}$, and the observed specific tunneling resistance and the broadening of the superconducting density of states (DOS) were reasonably low, giving us hope of also developing electronic coolers in the temperature range $1-3 \mathrm{~K}$ in the future. This was elaborated by numerical simulations, which demonstrated that a further lowering of the specific tunneling resistance of the junctions (in principle a straightforward process) would lead to a

a)Electronic mail: maasilta@jyu.fi sizeable electronic cooling at temperatures around $1.5-3 \mathrm{~K}$, despite the observed broadening of the superconducting density of states being higher than for typical Al junctions. In addition, if the broadening could be reduced to levels commonly seen for $\mathrm{Al}$, a truly wonderful device capable of reducing temperature from $1.2 \mathrm{~K}$ to $0.2 \mathrm{~K}$ would follow.

Tantalum nitride $\left(\mathrm{TaN}_{x}\right)$ is a material whose $\mathrm{T}_{C}$ has been shown to be tunable in thin films between 4 and $10.8 \mathrm{~K}$ by adjusting the growth parameters. ${ }^{12-15}$ Moreover, depending upon the amount of incorporated nitrogen $x, \mathrm{TaN}_{x}$ can be a superconductor, insulator, or a metal at low temperatures. ${ }^{12,16,17}$ In superconducting device applications, however, TaN has not been used widely. In its normal state, it has been used as a barrier material in superconductor-normal metalsuperconductor (SNS) Josephson junctions with NbN (Refs. 18-20) and NbTiN (Ref. 21) as the superconducting electrode materials. As a superconductor, the only device application so far has been for superconducting single photon detection, ${ }^{22}$ and notably, no tunnel junction devices have been reported before. Recently, we were able to grow high quality $\mathrm{TaN}_{x}$ thin films with $T_{\mathrm{C}}$ up to $\sim 8 \mathrm{~K}$ using a pulsed laser deposition (PLD) technique. ${ }^{13}$ Furthermore, we have already fabricated $\mathrm{NbN}_{x}$ based NIS junctions, using PLD for the growth of $\mathrm{NbN}_{x}$ films, and electron beam lithography (EBL), reactive ion etching (RIE), and shadow angle evaporation for the device fabrication, with an ex-situ thermally oxidized Al barrier. ${ }^{11}$ These two advances were combined here to develop $\mathrm{Cu}-\mathrm{AlO}_{x}-\mathrm{Al}-\mathrm{TaN}_{x} \mathrm{NIS}$ tunnel junctions.

First, $30 \mathrm{~nm}$ thick superconducting $\mathrm{TaN}_{x}$ films with $T_{\mathrm{C}}$ in the range $\sim 4.5-5 \mathrm{~K}$ were deposited on (100) oriented $\mathrm{MgO}$ single crystals using a PLD technique described in detail elsewhere. ${ }^{13}$ A typical temperature dependence of the resistance of such a bare $\mathrm{TaN}_{x}$ film is shown in Figure 1(a). $\mathrm{MgO}$ was chosen as the substrate because the films grown on it were shown to be monophase superconducting FCC (rocksalt), while in the films grown on oxidized silicon a coexisting non-superconducting hexagonal phase was also found. $^{13}$

The $\mathrm{TaN}_{x}$ films were patterned into $1 \mu \mathrm{m}$ wide electrodes and large contact pads by EBL and RIE. To make a more 


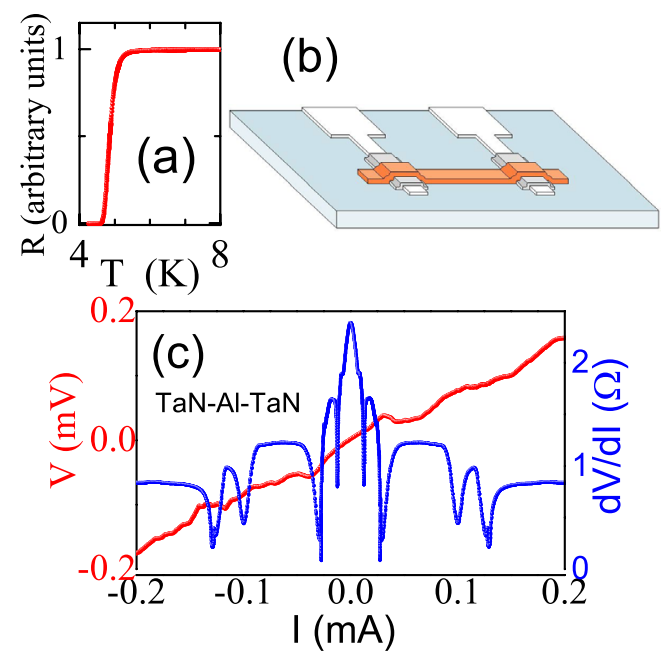

FIG. 1. Temperature dependence of the resistance of the bare $\operatorname{TaN}_{x}$ film, used for fabricating the $\mathrm{TaN}_{x}-\mathrm{Al}-\mathrm{AlO}_{x}$-Cu device, exhibiting superconducting transition at $\sim 5 \mathrm{~K}$. The ac bias current used was $10 \mu \mathrm{A}$. (b) A schematic of a fabricated device with two junctions. Blue: $\mathrm{MgO}$ substrate, White: TaN electrodes, Grey: Al/AlOx film, and Orange: $\mathrm{Cu}$ wire. (c) Bias current dependence of the voltage and differential resistance of a $\mathrm{TaN}_{x}-\mathrm{Al}-\mathrm{TaN}_{x} \mathrm{SNS}$ junction at $4.2 \mathrm{~K}$.

resistant etch mask, the $\mathrm{TaN}_{x}$ was first covered with a $50 \mathrm{~nm}$ thick evaporated $\mathrm{Cu}$ film, on top of which a $400 \mathrm{~nm}$ thick positive PMMA resist was spun, followed by EBL electrode patterning and removal of the $\mathrm{Cu}$ in the exposed regions with a chemical etch $\left(30 \% \mathrm{H}_{2} \mathrm{O}_{2}\right.$, glacial acetic acid, DI water 1:1:18). After that, the exposed $\mathrm{TaN}_{x}$ was etched by RIE using $\mathrm{CHF}_{3}, 50 \mathrm{sccm}$ and $\mathrm{O}_{2}, 5 \mathrm{sccm}$ at a power $100 \mathrm{~W}$ and pressure $55 \mathrm{mTorr}$, the PMMA removed, and finally the remaining $\mathrm{Cu}$ removed by another chemical etch step.

The electrode patterning was followed by the fabrication of three distinct types of devices using a second overlay EBL step and ultra-high vacuum (UHV) e-beam evaporation. For the first device type, a $40 \mathrm{~nm}$ thick, $0.5 \mu \mathrm{m}$ wide, and $15 \mu \mathrm{m}$ long aluminum cross strip was deposited across the electrodes, without any explicit attempt to form tunnel barriers. The purpose of this sample is to determine the quality of the $\mathrm{Al}-\mathrm{TaN}_{x}$ contact, as an unwanted native oxide barrier may exist on the surface of the $\mathrm{TaN}_{x}$ film. For the second device type, the method previously developed for the $\mathrm{NbN}_{x}$ NIS junction fabrication with $\mathrm{AlO}_{x}$ tunnel barriers has been used. ${ }^{11}$ First, $40 \mathrm{~nm}$ thick $\mathrm{Al}$ islands of size $6 \mu \mathrm{m} \times 6 \mu \mathrm{m}$ were evaporated on top the $\mathrm{TaN}_{x}$ electrodes, followed by in-situ oxidation at room temperature in 50 millibars of $\mathrm{O}_{2}$ for $4 \mathrm{~min}$, to grow the $\mathrm{AlO}_{x}$ tunnel barriers. Then, without breaking the vacuum, a $100 \mathrm{~nm}$ thick $\mathrm{Cu}$ strip of width $0.5 \mu \mathrm{m}$ was evaporated to form the connection between two $\mathrm{TaN}_{x}$ electrodes (separated by a distance of $15 \mu \mathrm{m}$ ) so that a series connection of two $\mathrm{Cu}-\mathrm{AlO}_{x}-\mathrm{Al}-\mathrm{TaN}_{x}$ NIS tunnel junctions (SINIS) is formed [Fig. 1(b)]. The third device type was identical with the second, except that no $\mathrm{Al}$ was deposited, and the $\mathrm{TaN}_{x}$ electrodes were directly oxidized in 400 millibars of $\mathrm{O}_{2}$ for $30 \mathrm{~min}$. The goal of this process is a SINIS device with a $\mathrm{Cu}-\mathrm{TaO}_{x}-\mathrm{TaN}_{x}$ tunnel junction structure. The typical junction dimensions were $\sim 1 \times 0.5 \mu \mathrm{m}^{2}$.

Since the $\mathrm{TaN}_{x}$ films come in contact with ambient atmosphere for prolonged periods of time during the process of fabrication, we investigated the effects of a possible native oxide barrier with the help of the first type of $\mathrm{TaN}_{x}-\mathrm{Al}-\mathrm{TaN}_{x}$ device. The measured voltage and differential resistance vs. current characteristics at $4.2 \mathrm{~K}$ are shown Figure 1(c). Clearly, the data shows that the contact resistance is low $<1 \Omega /$ junction (four orders of magnitude less than the tunneling resistances of the second and third type devices, as will be shown later), and that the general behavior is that of a good NS contact, although several resonance features are seen, possibly originating from multiple Andreev reflections. ${ }^{23}$ The resonance features were not observed in similar devices using NbN electrodes, ${ }^{11}$ however, the NS contact resistance in NbN devices was actually orders of magnitude higher for unknown reasons.

The current-voltage and conductance-voltage measurements for the second and third type devices were carried out using a $\mathrm{He}^{3}-\mathrm{He}^{4}$ dilution refrigerator. The measurement lines had three stages of filtering: pi-filters at $4.2 \mathrm{~K}, \mathrm{RC}$-filters at the base temperature, and microwave filtering ${ }^{24}$ between these two (Thermocoax cables of length $1.5 \mathrm{~m}$ ). For the measurement of conductance, a lock-in technique with a $0.04 \mathrm{mV}$ excitation voltage and $17 \mathrm{~Hz}$ frequency was used. In Figure 2, the current-voltage $(I-V)$ characteristics at various bath temperatures $T_{\text {Bath }}$ for a $\mathrm{TaN}_{x}-\mathrm{Al}-\mathrm{AlO}_{x}-\mathrm{Cu}$ based double junction SINIS device are shown in (a) log-linear and (b) linear scales, respectively, together with the corresponding theoretical fits based on the single-particle tunneling model $\frac{1}{e R_{\mathrm{T}}} \int_{-\infty}^{\infty} d \epsilon N_{S}(\epsilon)\left(f_{N}(\epsilon-e V)-f_{N}(\epsilon+e V)\right)$, where $f_{N}(\epsilon)$ is the Fermi function in $\mathrm{Cu}$ wire, and $N_{S}(\epsilon)$ is the normalized broadened superconducting quasiparticle DOS in the Dynes

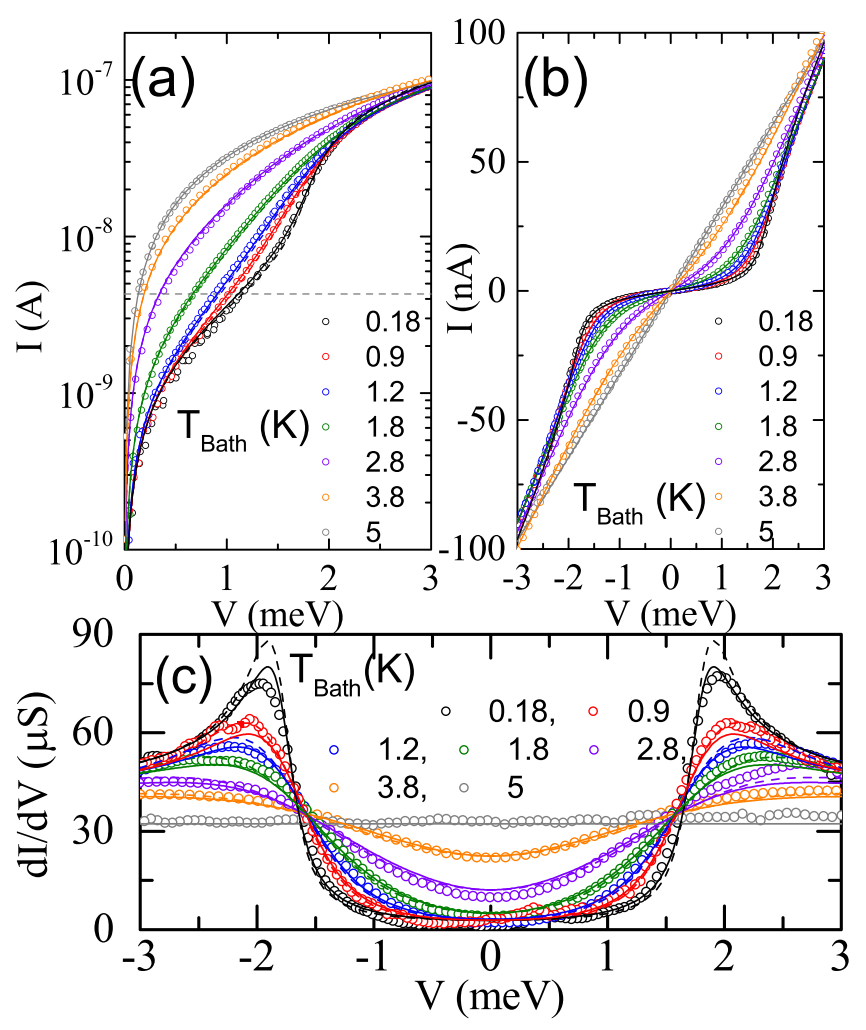

FIG. 2. Temperature dependence of the current-voltage characteristics of a double junction based $\mathrm{Cu}-\mathrm{AlO}_{x}-\mathrm{Al}-\mathrm{TaN}_{x}$ device at various $T_{\mathrm{Bath}}$ plotted in (a) log-linear and (b) linear scale. (c) Differential conductance characteristics corresponding to plots in (a). The open symbols denote the measured response, while the lines are the corresponding theoretical fits. The dashed and solid lines corresponds to calculations where the tunnelling resistance of individual junctions are assumed to be equal and unequal (proportions $66 \%$ and $34 \%$ ), respectively. The fitting parameters were $\Gamma / \Delta(0)=7 \times 10^{-2}$ and $R_{\mathrm{T}}$. The horizontal dashed line in (a) represents a constant current bias of $4.3 \mathrm{nA}$. 


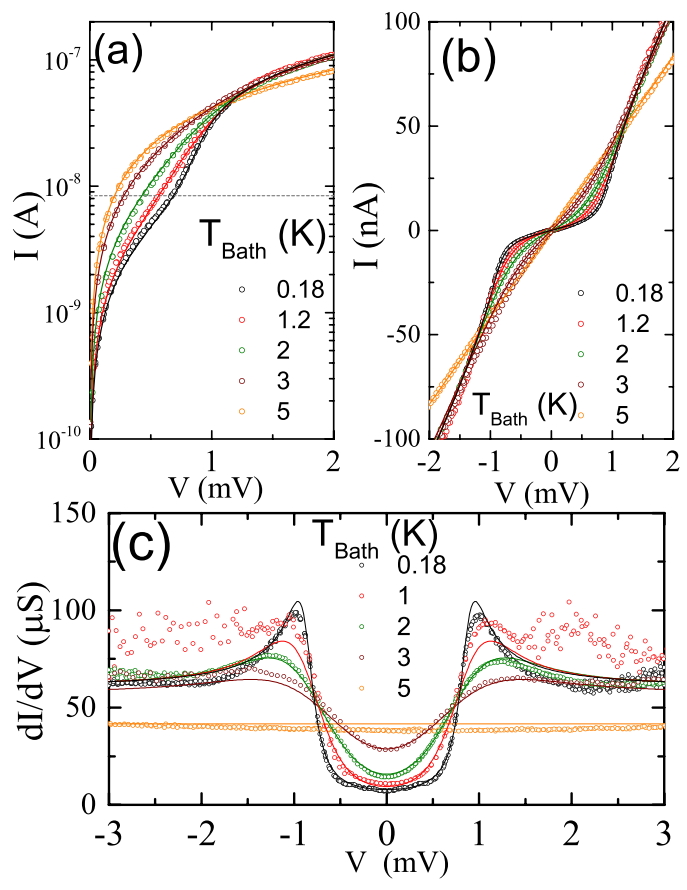

FIG. 3. Temperature dependence of the current-voltage characteristics of a single junction based $\mathrm{Cu}-\mathrm{TaO}_{x}-\mathrm{TaN}_{x}$ device at various $T_{\mathrm{Bath}}$ plotted in (a) $\log$ and (b) linear scale. (c) Differential conductance characteristics corresponding to plots in (a). The dots are the experimental data, while the solid lines are the corresponding theoretical fits. The fitting parameters were $\Gamma / \Delta(0)=0.13$ and $R_{\mathrm{T}}$. The horizontal dashed line in (a) represents a constant current bias of $8.4 \mathrm{nA}$.

model $^{1,25} N_{S}\left(\epsilon, T_{S}\right)=\left|\operatorname{Re}\left(\frac{\epsilon+i \Gamma}{\sqrt{(\epsilon+i \Gamma)^{2}-\Delta^{2}}}\right)\right|$, where $\Gamma$ is the parameter describing broadening and $\Delta$ is the superconducting gap. The corresponding conductance characteristics along with the theoretical fits are shown in Figure 2(c). For all these fits both the superconductor and normal metal temperatures $T_{S}$ and $T_{N}$ were set equal to $T_{\text {Bath. }}$. The dashed lines (most clearly visible for the lowest temperature data in (a) and (c)) assume that the tunneling resistances of the individual junctions are identical, while the solid lines assume non-identical tunneling resistances ${ }^{26}$ with proportions $66 \%$ and $34 \%$ of the total resistance. This asymmetry was directly measured with the help of a third NIS junction connected to the same normal metal electrode. The individual junction resistances can then be solved from the three measurements of the SINIS pairs. The agreement between the data and the simplest possible theory with two identical barriers is already very good at higher temperatures, where the effect of the asymmetry is weaker. However, at $0.18 \mathrm{~K}$, the symmetric model predicts a lower sub-gap current (visible at $V \sim 1.5 \mathrm{mV}$ ) than what is observed, and the non-symmetric theory can explain this increase. It is quite important to take into account the asymmetry at low $T$ : Simply fitting to the subgap conductance with $T_{N}$ as a free parameter would give $T_{N} \sim 0.5 \mathrm{~K}$. Such a high electronic overheating is unphysical, as it would require an excess heating power $>100$ pW (much higher than $\sim 10 \mathrm{fW}$ typically seen in our setup ${ }^{9}$ ), as estimated from the known electron-phonon interaction constant for $\mathrm{Cu}$ (Ref. 27) and the size of the normal metal island. The physical mechanism for the observed variability in $R_{\mathrm{T}}$ is unclear, although it has been suggested ${ }^{28}$ that it could result from grain-to-grain barrier variability.
From the fits, we also get the temperature dependence of the superconducting gap $\Delta$, which was seen to follow the simple BCS theory well, in contrast to the $\mathrm{NbN}_{x}$ based devices $^{11}$ which exhibited stronger modifications due to proximity effect. ${ }^{29}$ At $0.18 \mathrm{~K}$, the measured $\Delta$ was $\sim 0.9 \mathrm{meV}$, about four times higher than a typical $\mathrm{Al}$ film gap, indicating that the $\mathrm{Al}$ layer is well proximized by the $\mathrm{TaN}_{x}$. This value of $\Delta$ is almost the same as in the NbN NIS devices ${ }^{11}$ although $T_{\mathrm{C}}=5 \mathrm{~K}$ is less than half, an observation which is consistent with the fact that the contact resistance between $\mathrm{TaN}_{x}$ and $\mathrm{Al}$ is much lower. All the theory fits to the $I-V$ and conductance curves were obtained with a broadening parameter $\Gamma / \Delta(0)=7 \times 10^{-2} \quad[\Delta(0)=\Delta(T=0)]$, a value which is slightly higher than the smallest value observed in the $\mathrm{NbN}_{x}$ devices, $\Gamma_{\mathrm{NbN}} / \Delta_{\mathrm{NbN}}=2.4 \times 10^{-2}$. Similar to the $\mathrm{NbN}$ case, strong coupling theory did not fit the data well (not shown). Finally, the total $R_{\mathrm{T}}$ of this device was, surprisingly, found to evolve with temperature, from $\sim 31 \mathrm{k} \Omega$ at $5 \mathrm{~K}$ to $\sim 26.5 \mathrm{k} \Omega$ at $3.8 \mathrm{~K}$ and $\sim 24.5 \mathrm{k} \Omega$ at still lower temperatures. This translates to a specific junction resistance $r_{\mathrm{S}}$ of $\sim 6.5 \mathrm{k} \Omega \mu \mathrm{m}^{2}$, which is about two orders of magnitude smaller than that in the $\mathrm{NbN}_{x}$ devices fabricated in a similar manner, but still about three-ten times higher than that of typical high power $\mathrm{Cu}-\mathrm{AlO}_{x}$ - $\mathrm{Al}$ tunnel junction coolers. ${ }^{4,5}$

For the devices of the third type $\left(\mathrm{TaN}_{x}-\mathrm{TaO}_{x}-\mathrm{Cu}\right)$, the yield was quite low-most of them were shorts. However, some were tunnel junctions. In Figures 3(a) and 3(b), the current-voltage $(I-V)$ characteristics at various $T_{\text {Bath }}$ of such a $\mathrm{TaN}_{x}-\mathrm{TaO}_{x}-\mathrm{Cu}$ single NIS junction are shown in (a) log-linear and (b) linear scale, respectively, together with the corresponding theoretical fits. The measured and theoretical conductance curves are shown in Figure 3(c). From the theoretical fits, the obtained value of $\Gamma / \Delta(0)$ and $\Delta(0)$ were 0.13 and $0.87 \mathrm{meV}$, respectively, with $T_{\mathrm{C}}=4.5 \mathrm{~K}$ being the measured value of the $\mathrm{TaN}_{x}$ film. Here, the obtained value of $R_{\mathrm{T}}$ evolved with temperature even more strongly, from $\sim 24 \mathrm{k} \Omega$ at $5 \mathrm{~K}$ to $\sim 16.5 \mathrm{k} \Omega$ below $5 \mathrm{~K}$. The origin of this temperature dependence of $R_{\mathrm{T}}$ is unclear to us at the moment. The largest change seems to be correlated with the transition to the superconducting state, but some temperature dependence seems to be left even at temperatures much below $T_{\mathrm{C}}$. Although the $\Delta$ values of $\mathrm{TaN}_{x}-\mathrm{TaO}_{x}-\mathrm{Cu}$ and $\mathrm{TaN}_{x}-\mathrm{Al}_{-}-\mathrm{AlO}_{x}-\mathrm{Cu}$ junctions are almost identical, the low yield and the larger value of $\Gamma$ of the latter render them unfit for real device applications.

Figure 4 shows the thermometric response in the usual measurement configuration where the NIS junction device is current biased, and its voltage $(V)$ response is measured as a function of $T_{\mathrm{Bath}}$, of the (a) double junction $\mathrm{TaN}_{x}-\mathrm{Al}_{-}-\mathrm{AlO}_{x}-\mathrm{Cu}$ device and (b) single junction $\mathrm{TaN}_{x}-\mathrm{TaO}_{x}$-Cu device. For both devices, the measured temperature sensitivity was $\sim 0.14 \mathrm{mV} / \mathrm{K} /$ junction from $T_{\mathrm{C}}$ down to $\sim 0.5 \mathrm{~K}$, as expected from theory, but at the lowest temperatures there is a saturation and even a curious downturn of the voltage. This downturn cannot be explained by any theory where $R_{\mathrm{T}}$ is temperature independent for such low bias (sub-gap) values, ${ }^{26}$ and thus the thermometry data confirms the picture of changing $R_{\mathrm{T}}$, as can be seen from the representative theory curves.

Having obtained the values for $\Gamma$ and $r_{\mathrm{S}}$ for the TaN NIS devices, we should compare them with previous results using other superconductors. In Table I, we have complied 

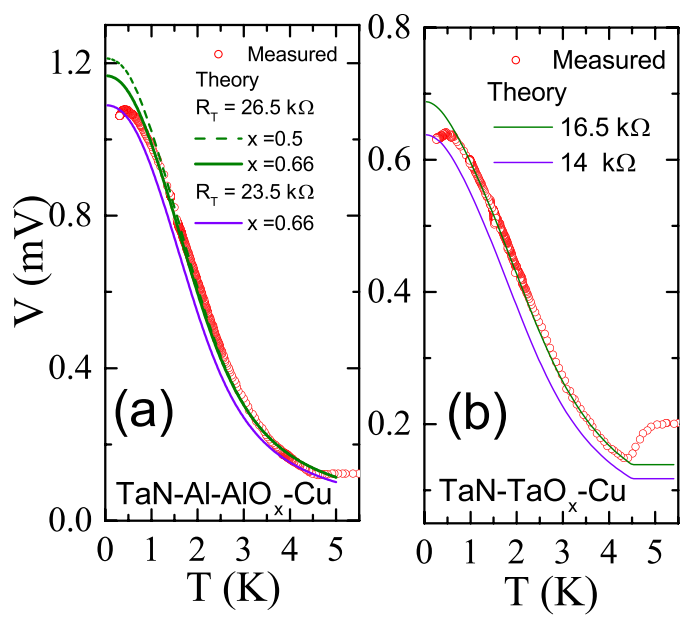

FIG. 4. Thermometry characteristics of the (a) $\mathrm{Cu}-\mathrm{AlO}_{x}-\mathrm{Al}-\mathrm{TaN}_{x}$ junction pair biased with a constant current of $\sim 4.3 \mathrm{nA}$ and (b) a single $\mathrm{Cu}-\mathrm{TaO}_{x}-\mathrm{TaN}_{x}$ junction biased with $\sim 8.4 \mathrm{nA}$. The dots are the experimental data, while the lines indicate the corresponding theoretical fits, assuming a simple one particle tunnelling model with BCS temperature dependence of the superconducting gap, for various cases of $R_{\mathrm{T}}$. In (a), dashed line, symmetric $R_{\mathrm{T}}=26.5 \mathrm{k} \Omega$, solid green line, asymmetric $R_{\mathrm{T}}=26.5 \mathrm{k} \Omega$ with proportions $66 \%$ and $34 \%$ of the two junction resistances, solid purple line, $R_{\mathrm{T}}=23.5 \mathrm{k} \Omega$, same asymmetry. In (b), solid green line, $R_{\mathrm{T}}=16.5 \mathrm{k} \Omega$, solid purple line, $R_{\mathrm{T}}=14 \mathrm{k} \Omega$.

results from our lab, fabricated in the same chamber and with fairly similar oxidation parameters. The parameters for $\mathrm{TaN}_{x}-\mathrm{Al}-\mathrm{AlO}_{x}-\mathrm{Cu}$ junctions seem to be comparable to the earlier results for $\mathrm{Nb}-\mathrm{Al}-\mathrm{AlO}_{x}$-Cu junctions. The biggest difference to the standard $\mathrm{Al}-\mathrm{AlO}_{x}-\mathrm{Cu}$ junction technology is the much larger value of the broadening parameter $\Gamma$. The $\mathrm{NbN}$ junctions do not seem as promising for cooling as TaN junctions due to the high specific junctions resistances. The DOS broadening seen in our $\mathrm{Al}-\mathrm{AlO}_{x}$-Cu junctions is comparable to the results by other labs. ${ }^{28,30,31}$ However, if extreme measures are taken to reduce environmental radiation coupling to the junction, much lower broadening has been demonstrated in $\mathrm{Al}-\mathrm{AlO}_{x}-\mathrm{Cu}$ junctions, ${ }^{30,32}$ explained by photon-assisted tunneling. According to that picture $\Gamma / \Delta \sim$ $1 / \Delta$, which suggests that the broadening in our higher gap junctions $(\mathrm{Nb}, \mathrm{NbN}, \mathrm{TaN})$ is due to some other mechanism.

Finally, to answer better whether TaN based NIS junctions hold promise for cooling applications, we also carried out some numerical simulations. To give an example, all calculations assumed a SINIS device with $T_{\mathrm{C}}=5 \mathrm{~K}$ and $\Delta=0.9 \mathrm{meV}$, and a $\mathrm{Cu}$ normal metal island of thickness

TABLE I. Broadening of the density of states $\Gamma / \Delta(0)$, specific tunneling resistance $r_{\mathrm{S}}$, energy gap at low temperature $\Delta(0)$, and critical temperature $T_{\mathrm{C}}$ for various types of junctions. All these junctions were oxidized in the same physical chamber under fairly identical oxidation conditions.

\begin{tabular}{|c|c|c|c|c|c|}
\hline Junction & $\frac{\Gamma}{\Delta(0)}$ & $\begin{array}{c}r_{\mathrm{S}} \\
\left(\mathrm{k} \Omega \mu \mathrm{m}^{2}\right)\end{array}$ & $\begin{array}{c}\Delta(0) \\
(\mathrm{meV})\end{array}$ & $\begin{array}{l}T_{\mathrm{C}} \\
(\mathrm{K})\end{array}$ & References \\
\hline $\mathrm{Al}-\mathrm{AlO}_{x}-\mathrm{Cu}$ & $1-5 \times 10^{-4}$ & $1-2$ & 0.21 & 1.4 & 9 \\
\hline $\mathrm{Nb}-\mathrm{Al}-\mathrm{AlO}_{x}-\mathrm{Cu}$ & $5 \times 10^{-2}$ & 10 & 1.0 & 6 & 10 \\
\hline $\mathrm{NbN}_{x}-\mathrm{Al}-\mathrm{AlO}_{x}-\mathrm{Cu}$ & $2-4 \times 10^{-2}$ & $630-770$ & 1.1 & 10.8 & 11 \\
\hline $\mathrm{NbN}_{x}-\mathrm{NbO}_{x}-\mathrm{Cu}$ & 0.2 & 40 & 1.1 & 10.8 & 11 \\
\hline $\mathrm{TaN}_{x}-\mathrm{Al}-\mathrm{AlO}_{x}-\mathrm{Cu}$ & $7 \times 10^{-2}$ & 6.5 & 0.9 & 5 & \\
\hline $\mathrm{TaN}_{x}-\mathrm{TaO}_{x}-\mathrm{Cu}$ & 0.13 & 8 & 0.9 & 4.5 & \\
\hline
\end{tabular}

$30 \mathrm{~nm}$, with a lateral size the same as the total junction area $A$. Electron-phonon interaction limited heat flow out of the island was also assumed, which is the typical situation for junctions on bulk substrates, ${ }^{2,9}$ leading to heat balance $P_{\text {cool }}=\Sigma \Omega\left(T_{\text {bath }}^{5}-T_{N}^{5}\right)$, where $P_{\text {cool }}$ is the cooling power of the junctions that can be calculated when junctions parameters are known, ${ }^{2,9} \Omega$ is the normal metal volume, and $\Sigma$ is the electron-phonon coupling constant. A typical value for $\Sigma=2 \times 10^{9} \mathrm{~W} /\left(\mathrm{m}^{3} \mathrm{~K}^{5}\right)$ in $\mathrm{Cu}$ was used. ${ }^{27}$ Since $P_{\text {cool }} \propto A$ and $\Omega \propto A$, the results shown here are independent of $A$, and therefore we use the specific junction resistance $r_{\mathrm{S}}$ as parameter. In Figure 5(a), we show the expected decrease of $T_{N}$ below $T_{\text {Bath }}$, as a function of $\Gamma$ and $T_{\text {Bath }}$ for the value of specific junction resistance observed in the experiment $r_{\mathrm{S}}=6.5 \mathrm{k} \Omega \mu \mathrm{m}^{2}$. We find that a bit of cooling is expected at low $T_{\text {Bath }} \sim 0.2-0.3 \mathrm{~K}$ if $\Gamma / \Delta(0)$ could be lowered to values $<10^{-3}$. However, at that temperature range $\mathrm{Al}$ coolers perform better. On the other hand, if the value of $r_{\mathrm{S}}$ is lowered, as shown in Fig. $5(\mathrm{~b})$, but $\Gamma / \Delta(0)$ is fixed at the observed value $7 \times 10^{-2}$, a fair amount of cooling (up to $0.3 \mathrm{~K}$ ) at high $T_{\text {Bath }} \sim 2-3 \mathrm{~K}$ is possible when $r_{\mathrm{S}}<10 \Omega \mu \mathrm{m}^{2}$. Even this would fall far short from the ultimate goal to cool the metal island from $1.2 \mathrm{~K}$ to $0.3 \mathrm{~K}$. In order to achieve such a large magnitude in cooling, a concomitant reduction in $\Gamma / \Delta(0)$ of these $\mathrm{TaN}_{x}$ devices is also necessary. As shown if Fig. 5(c), if $\Gamma / \Delta(0)$ could be lowered to $1 \times 10^{-4}$ (typical for Al devices), then for $r_{\mathrm{S}}<100 \Omega \mu \mathrm{m}^{2}$ such a large cooling would be theoretically possible. Interestingly, these kind of values for $\Gamma$ and $r_{\mathrm{S}}$ have been obtained experimentally for $\mathrm{Al}-\mathrm{AlO}_{x}-\mathrm{Cu}$ junctions.

In conclusion, we have demonstrated the application potential of normal metal-insulator-superconductor tunnel junction devices with $\mathrm{TaN}$ as the superconductor. The electrical characteristics of these devices follow the simple oneparticle tunneling model, and the superconducting gap exhibit a BCS type temperature dependence. We also demonstrated sensitive thermometry between 0.5 and $5 \mathrm{~K}$, where the lower limit was shown to be caused by an unexpected

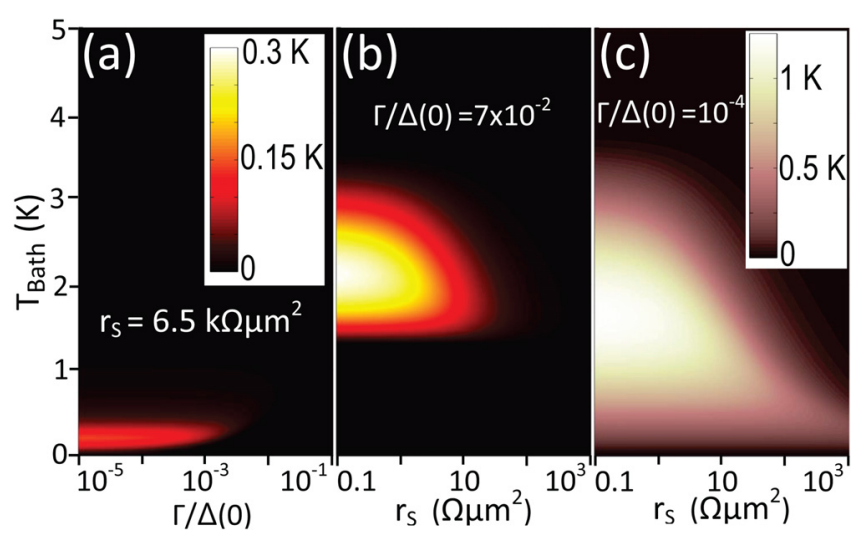

FIG. 5. Calculated electronic cooling $\left(T_{\text {Bath }}-T_{N}\right)$ for several cases, with electron-phonon limited heat transport and $\mathrm{Cu}$ as normal metal. Only regions where net cooling occur $\left(T_{\mathrm{Bath}} \geq T_{N}\right)$ are shown. (a) Cooling as a function of $T_{\text {Bath }}$ and $\Gamma / \Delta(0)$ for $\mathrm{r}_{S}=6.5 \mathrm{k} \Omega \mu \mathrm{m}^{2}$. In (b) and (c), calculated cooling as a function of $T_{\mathrm{B}}$ ath and $r_{\mathrm{S}}$ for (b) $\Gamma / \Delta(0)=7 \times 10^{-2}$ and (c) $\Gamma / \Delta(0)=1 \times 10^{-4}$. The color bars indicates the magnitude of cooling. The color bar in the inset of (a) serves as the scale for both (a) and (b). It can be seen from (c) that for a device with $\Gamma / \Delta(0)=1 \times 10^{-4}$ and $r_{\mathrm{S}} \sim 10 \Omega \mu \mathrm{m}^{2}$, the expected cooling at $1.2 \mathrm{~K}$ is $\sim 1 \mathrm{~K}$. For all simulations $T_{C}=5 \mathrm{~K}$ and $\Delta=0.9 \mathrm{meV}$. 
temperature variability of the tunneling resistance. The measured effective broadening of the superconducting density of states and the specific tunneling resistance of these devices were just high enough to inhibit any electronic cooling. However, as we showed theoretically, a realistic reduction of these parameters for TaN devices would lead to a dramatic breakthrough in the development of practical electronic coolers for $1 \mathrm{~K}$ temperature range. Future efforts need to be especially focused on understanding the broadening of the superconducting density of states and how to reduce it.

This research has been supported by Academy of Finland Project No. 260880. We thank A. Torgovkin for help with low temperature measurements.

${ }^{1}$ F. Giazotto, T. T. Heikkilä, A. Luukanen, A. M. Savin, and J. P. Pekola, Rev. Mod. Phys. 78, 217 (2006).

${ }^{2}$ J. T. Muhonen, M. Meschke, and J. P. Pekola, Rep. Prog. Phys. 75, 046501 (2012).

${ }^{3}$ M. Nahum and J. M. Martinis, Appl. Phys. Lett. 63, 3075 (1993).

${ }^{4}$ P. J. Lowell, G. C. O'Neil, J. M. Underwood, and J. N. Ullom, Appl. Phys. Lett. 102, 082601 (2013).

${ }^{5}$ H. Q. Nguyen, T. Aref, V. J. Kauppila, M. Meschke, C. B. Winkelmann, H. Courtois, and J. P. Pekola, New J. Phys. 15, 085013 (2013).

${ }^{6}$ A. Luukanen, M. M. Leivo, J. K. Suoknuuti, A. J. Manninen, and J. P. Pekola, J. Low Temp. Phys. 120, 281 (2000).

${ }^{7}$ A. M. Clark, N. A. Miller, A. Williams, S. T. Ruggiero, G. C. Hilton, L. R. Vale, J. A. Beall, K. D. Irwin, and J. N. Ullom, Appl. Phys. Lett. 86, 173508 (2005).

${ }^{8}$ N. A. Miller, G. C. O’Neil, J. A. Beall, G. C. Hilton, K. D. Irwin, D. R. Schmidt, L. R. Vale, and J. N. Ullom, Appl. Phys. Lett. 92, 163501 (2008).

${ }^{9}$ P. J. Koppinen and I. J. Maasilta, Phys. Rev. Lett. 102, 165502 (2009).

${ }^{10}$ M. R. Nevala, S. Chaudhuri, J. Halkosaari, J. T. Karvonen, and I. J. Maasilta, Appl. Phys. Lett. 101, 112601 (2012).

${ }^{11}$ S. Chaudhuri, M. R. Nevala, and I. J. Maasilta, Appl. Phys. Lett. 102, 132601 (2013).

${ }^{12}$ F. M. Kilbane and P. S. Habig, J. Vac. Sci. Technol. 12, 107 (1975).
${ }^{13}$ S. Chaudhuri, I. J. Maasilta, L. Chandernagor, M. Ging, and M. Lahtinen, J. Vac. Sci. Technol., A 31, 061502 (2013).

${ }^{14}$ K. Reichelt, W. Nellen, and G. Mair, J. Appl. Phys. 49, 5284 (1978).

${ }^{15}$ K. Ilin, M. Hofherr, D. Rall, M. Siegel, A. Semenov, A. Engel, K. Inderbitzin, A. Aeschbacher, and A. Schilling, J. Low Temp. Phys. 167, 809 (2012).

${ }^{16}$ H. Nie, S. Xu, S. Wang, L. You, Z. Yang, C. Ong, J. Li, and T. Liew, Appl. Phys. A 73, 229 (2001).

${ }^{17}$ L. Yu, C. Stampfl, D. Marshall, T. Eshrich, V. Narayanan, J. M. Rowell, N. Newman, and A. J. Freeman, Phys. Rev. B 65, 245110 (2002).

${ }^{18}$ A. B. Kaul, S. R. Whiteley, T. Van Duzer, L. Yu, N. Newman, and J. M. Rowell, Appl. Phys. Lett. 78, 99 (2001).

${ }^{19}$ R. Setzu, E. Baggetta, and J. C. Villegier, J. Phys.: Conf. Ser. 97, 012077 (2008).

${ }^{20}$ M. Nevala, I. Maasilta, K. Senapati, and R. Budhani, IEEE Trans. Appl. Supercond. 19, 253 (2009).

${ }^{21}$ L. Yu, R. Gandikota, R. K. Singh, L. Gu, D. J. Smith, X. Meng, T. Zeng, X. Van Duzer, J. M. Rowell, and N. Newman, Supercond. Sci. Technol. 19, 719 (2006).

${ }^{22}$ A. Engel, A. Aeschbacher, K. Inderbitzin, A. Schilling, K. Ilin, M. Hofherr, M. Siegel, A. Semenov, and H.-W. Hübers, Appl. Phys. Lett. 100, 062601 (2012).

${ }^{23}$ J. C. Cuevas, J. Hammer, J. Kopu, J. K. Viljas, and M. Eschrig, Phys. Rev. B 73, 184505 (2006).

${ }^{24}$ A. B. Zorin, Rev. Sci. Instrum. 66, 4296 (1995).

${ }^{25}$ R. C. Dynes, J. P. Garno, G. B. Hertel, and T. P. Orlando, Phys. Rev. Lett. 53, 2437 (1984).

${ }^{26}$ S. Chaudhuri and I. J. Maasilta, Phys. Rev. B 85, 014519 (2012).

${ }^{27}$ J. T. Karvonen, L. J. Taskinen, and I. J. Maasilta, J. Low Temp. Phys. 149, 121 (2007).

${ }^{28}$ T. Greibe, M. P. V. Stenberg, C. M. Wilson, T. Bauch, V. S. Shumeiko, and P. Delsing, Phys. Rev. Lett. 106, 097001 (2011).

${ }^{29}$ A. A. Golubov, E. P. Houwman, J. G. Gijsbertsen, V. M. Krasnov, J. Flokstra, H. Rogalla, and M. Y. Kupriyanov, Phys. Rev. B 51, 1073 (1995).

${ }^{30}$ J. P. Pekola, V. F. Maisi, S. Kafanov, N. Chekurov, A. Kemppinen, Y. A. Pashkin, O.-P. Saira, M. Möttönen, and J. S. Tsai, Phys. Rev. Lett. 105, 026803 (2010).

${ }^{31}$ G. C. O'Neil, P. J. Lowell, J. M. Underwood, and J. N. Ullom, Phys. Rev. B 85, 134504 (2012).

${ }^{32}$ O.-P. Saira, A. Kemppinen, V. F. Maisi, and J. P. Pekola, Phys. Rev. B 85, 012504 (2012). 